Check for updates

Cite this: J. Mater. Chem. B, 2017, 5, 3418

DOI: $10.1039 / c 7 t b 90054 f$

rsc.li/materials-b

\section{Correction: Photocrosslinkable, biodegradable hydrogels with controlled cell adhesivity for prolonged siRNA delivery to hMSCs to enhance their osteogenic differentiation}

\author{
Minh Khanh Nguyen, ${ }^{a}$ Alexandra McMillan, ${ }^{b}$ Cong Truc Huynh, ${ }^{a}$ Daniel S. Schapira ${ }^{a}$ \\ and Eben Alsberg*acd
}

Correction for 'Photocrosslinkable, biodegradable hydrogels with controlled cell adhesivity for prolonged siRNA delivery to hMSCs to enhance their osteogenic differentiation' by Minh Khanh Nguyen et al., J. Mater. Chem. B, 2017, 5, 485-495.

The authors regret the following errors in the original manuscript.

1. Old text in "Hydrogel preparation" section:

The hydrogels were formed upon exposure of DEX-MAES solutions to $320-500 \mathrm{~nm}$ UV light at $2.5 \mathrm{~mW} \mathrm{~cm}^{-2}$ for 2 min using an Omnicure S1000 UV Spot Cure System (Lumen Dynamics Group, Mississauga, Ontario, Canada).

Correction (bold text was changed and/or added):

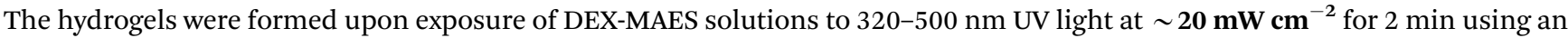
Omnicure S1000 UV Spot Cure System (Lumen Dynamics Group, Mississauga, Ontario, Canada). Note: UV intensity was measured as $2.5 \mathrm{~mW} \mathrm{~cm}^{-2}$ during the performance of this study using a UV meter that was later determined to be malfunctioning and supplying erroneous values. Once this was discovered, the UV intensity was remeasured to be $\sim 20 \mathrm{~mW} \mathrm{~cm}^{-2}$ based on the original instrument experimental setup parameters using a new and properly functioning UV meter. Although there may have been slight changes within the Omnicure UV instrument over time such as decreased bulb output, the updated UV intensity of $\sim 20 \mathrm{~mW} \mathrm{~cm}$ more accurately represents that actually used and replaces the previously reported value throughout this paper.

2. Old text in "Rheology" section:

PBS solutions of DEX-MAES containing $0.05 \% \mathrm{w} / \mathrm{v}$ Igracure D-2959 photoinitiator were placed between a glass plate and a quartz plate separated by two $0.75 \mathrm{~mm}$ spacers followed by photocrosslinking via the application of UV light $\left(2.5 \mathrm{~mW} \mathrm{~cm}^{-2}\right)$ for 2 min.

Correction (bold text was changed):

PBS solutions of DEX-MAES containing $0.05 \% \mathrm{w} / \mathrm{v}$ Igracure D-2959 photoinitiator were placed between a glass plate and a quartz plate separated by two $0.75 \mathrm{~mm}$ spacers followed by photocrosslinking via the application of UV light $\left(\sim 20 \mathbf{~ m W ~} \mathbf{~ m}^{-2}\right)$ for $2 \mathrm{~min}$.

3. Old text in "Viability and osteogenic differentiation of encapsulated hMSCs in hydrogels" section:

The hydrogels were cultured in 24 well plates with $0.5 \mathrm{ml}$ of osteogenic media $(10 \mathrm{mM}$-glycerophosphate (CalBiochem, Billerica, MA), $50 \mu \mathrm{M}$ ascorbic acid (Wako USA, Richmond, VA), $100 \mathrm{nM}$ dexamethasone (MP Biomedicals, Solon, OH) and $100 \mathrm{ng} \mathrm{ml}^{-1}$ BMP-2 (Department of Developmental Biology, University of Würzburg, Germany)).

Correction (bold text was added):

The hydrogels were cultured in 24 well plates with $0.5 \mathrm{ml}$ of osteogenic media (DMEM-LG supplemented with 10\% FBS, $1 \%$ penicillin/streptomycin (Fisher Scientific), $10 \mathrm{mM} \beta$-glycerophosphate (CalBiochem, Billerica, MA), $50 \mu \mathrm{M}$ ascorbic acid (Wako USA, Richmond, VA), $100 \mathrm{nM}$ dexamethasone (MP Biomedicals, Solon, OH) and $100 \mathrm{ng} \mathrm{ml}^{-1}$ BMP-2 (Department of Developmental Biology, University of Würzburg, Germany)).

\footnotetext{
${ }^{a}$ Department of Biomedical Engineering, Case Western Reserve University, 10900 Euclid Ave., Cleveland, Ohio 44106, USA. E-mail: eben.alsberg@case.edu

${ }^{b}$ Department of Pathology, Case Western Reserve University, 10900 Euclid Ave., Cleveland, Ohio 44106, USA

${ }^{c}$ Department of Orthopaedic Surgery, Case Western Reserve University, 10900 Euclid Ave., Cleveland, Ohio 44106, USA

${ }^{d}$ National Center for Regenerative Medicine, Division of General Medical Sciences, Case Western Reserve University, 10900 Euclid Ave., Cleveland, Ohio 44106, USA
} 
4. Fig. 6C in the original manuscript is a mistaken duplicate of Fig. 6A. The correct version of Fig. 6C is shown below:

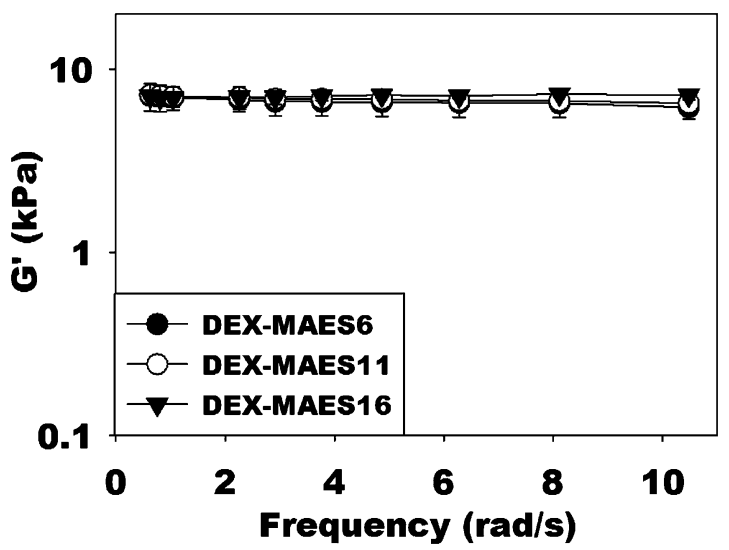

The Royal Society of Chemistry apologises for these errors and any consequent inconvenience to authors and readers. 